\title{
The Solubility of Cucurbit[5]uril in Pure Water and Hydrochloric Acid at Different Temperature and Some Relative Thermodynamic Functions
}

\author{
Hongyan $\mathrm{LU}^{1,}$, Jiarong $\mathrm{LI}^{1, \mathrm{~b}}$ \\ ${ }^{1}$ School of Chemical \& Environmental Engineering, Beijing Institute of Technology, Beijing, 100081, \\ China \\ aemail: luhongyan1202@163.com, bemail:jrli@bit.edu.cn
}

Keywords: solubility; cucurbit[5]uril; enthalpy; entropy; Gibbs free energy

\begin{abstract}
The solubility of cucurbit[5]uril in pure water and different concentration of hydrochloric acid aqueous solution( $\mathrm{HCl}: \mathrm{H}_{2} \mathrm{O}=10: 110,15: 105,20: 100,40: 80$ ) were investigated between $283.15 \mathrm{~K}$ and $323.15 \mathrm{~K}$ at atmospheric pressure by using the UV-vis spectrophotometer method. The solubility of $\mathrm{CB}[5]$ increased with the increasing of temperature, and a new phenomenon was found that the solubility of $\mathrm{CB}[5]$ increased with increasing acid concentration below $298 \mathrm{~K}$ and the solubility of $\mathrm{CB}[5]$ reduced with increasing acid concentration above $305 \mathrm{~K}$. Thermodynamic functions (entropy and enthalpy of dissolution) for the $\mathrm{CB}[5]$ solutions were determined with the Van't Hoff equation, and the Gibbs free energy was also calculated.
\end{abstract}

\section{Introduction}

In 1905, Behrend and coworkers synthesized cucurbituril(CB[n]) from condensation of glycoluril and formaldehyde under strongly acidic conditions [1]. A series of homologues and derivatives $(\mathrm{CB}[\mathrm{n}] \mathrm{n}=5,6,7,8,10, t \mathrm{CB}[14]$ and $i \mathrm{CB}[\mathrm{n}] \mathrm{n}=5,6)$ [2-7] have been separated and purified since $\mathrm{CB}[6]$ was first characterized in 1981 [8]. Due to the unique hydrophobic cavity and hydrophilic portal of cucurbiturils, the particular properties of cucurbiturils is the focus of attention in supramolecular chemistry. Over the past decade, there have been reported broad application of $\mathrm{CB}[\mathrm{n}$ ] in the fields of medication [9], molecular machines [10], sensing ensembles [11], marerials [12], and so on.

In a series of cucurbituril analogs and derivatives, $\mathrm{CB}[5]$ and $\mathrm{CB}$ [7] have higher solubility in common solvents than $\mathrm{CB}[6]$ and $\mathrm{CB}$ [8].[2] [4] [13-17]. Thus there are a lot of studies on $\mathrm{CB}$ [5] and $\mathrm{CB}[7]$ in recent years. For example, some new studies have been focused on complexation with Metal ion (K, Na, lanthanides, copper(II), uranyl ions, hydrated $\mathrm{Cs}^{+}$ions and ect. ) [18-23]. Researchers studied the synthesis and crystal structure of a one-dimensional heterometallic coordination polymer based on $\mathrm{CB}$ [5] [24]. There also some research studied the use of $\mathrm{CB}$ [5] for improving the photostability and/or the storage stability, increasing the absorbance and/or fluorescence quantum, or altering the fluorescence spectrum of fluorescence dye compounds [25].

As known from the reference, some studies on the solubility of $\mathrm{CB}[\mathrm{n}](\mathrm{n}=5,6,7,8)$ in some different concentration of hydrochloric acid aqueous solution, formic acid, or acetic acid at room temperature [13-17], have been reported. Nevertheless, there is no report about the solubility data of $\mathrm{CB}[\mathrm{n}]$ in different temperature, which is very important in the preparation, purification and application of $\mathrm{CB}[\mathrm{n}]$. In this study, the solubility of $\mathrm{CB}[5]$ in pure water and different concentration of hydrochloric acid aqueous solution were measured using $U V-$ vis spectrophotometer method ${ }^{28}$ at temperatures ranging from 283.15 to $323.15 \mathrm{~K}$. The enthalpy, entropy, and Gibbs free energy of solution for $\mathrm{CB}[5]$ was calculated from the experiment data. 


\section{Results and Discussion}

The solubility of $\mathrm{CB}[5]$ in pure water and different concentration of hydrochloric acid aqueous solution were measured at the temperatures of $283.15 \mathrm{~K}, 293.15 \mathrm{~K}, 303.15 \mathrm{~K}, 313.15 \mathrm{~K}$, and 323.15 $\mathrm{K}$, respectively. And the data were listed in Table1.

Table 1 . The solubility of CB[5] in pure water and different concentration of hydrochloric acid aqueous at different temperatures ${ }^{\mathrm{a}}$

\begin{tabular}{cccccc}
\hline $\mathrm{HCl}: \mathrm{H}_{2} \mathrm{O}(\mathrm{V}: \mathrm{V})$ & $0: 120$ & $10: 110$ & $15: 105$ & $20: 100$ & $40: 80$ \\
\hline $283.15 \mathrm{~K}$ & 0.6893 & 1.0672 & 1.3079 & 1.0102 & 1.6072 \\
\hline $293.15 \mathrm{~K}$ & 1.6143 & 1.8686 & 2.4736 & 3.0388 & 2.5549 \\
\hline $303.15 \mathrm{~K}$ & 9.8475 & 6.5271 & 4.7676 & 5.8784 & 4.1542 \\
\hline $313.15 \mathrm{~K}$ & 11.5775 & 13.0541 & 6.2102 & 8.2178 & 5.4417 \\
\hline $323.15 \mathrm{~K}$ & 24.8328 & 15.0381 & 11.4908 & 12.8392 & 8.7655 \\
\hline
\end{tabular}

${ }^{\mathrm{a}}$ The unit of the $\mathrm{CB}[5]$ solubility is $10^{-2} \mathrm{~mol} \cdot \mathrm{L}^{-1}$.

The mole fraction $(x)$ solubilities of $\mathrm{CB}[5]$ in pure water and different concentration of hydrochloric acid aqueous solution in the range of 273.15 to $323.15 \mathrm{~K}$ are listed in Figure 1
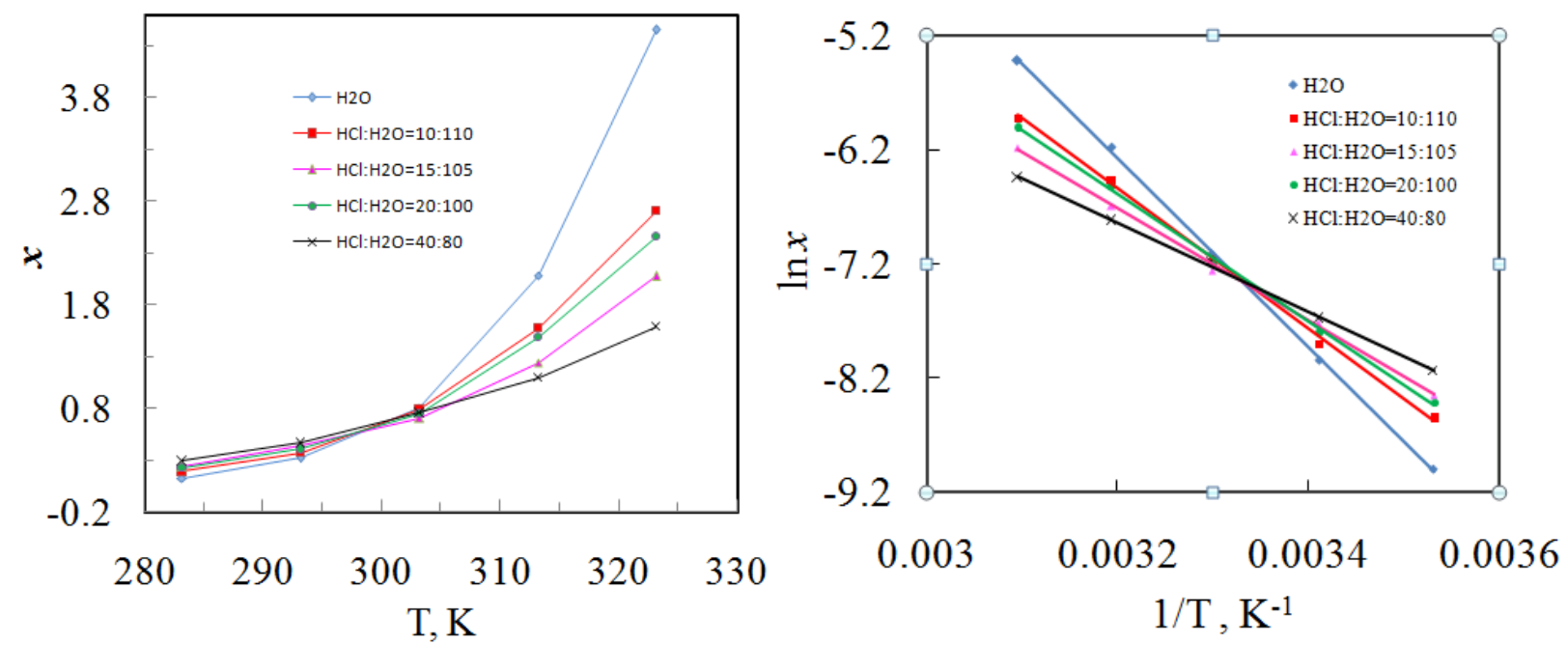

Figure 1. (a)Mole fraction solubility of $\mathrm{CB}$ [5] from $283.15 \mathrm{~K}$ to $323.15 \mathrm{~K}$ in different solvents (b) Temperature dependence of $\ln (x)$ of $\mathrm{CB}[5]$ in different solvents: $\rightarrow \mathrm{H}_{2} \mathrm{O}, \quad \quad \mathrm{HCl}: \mathrm{H}_{2} \mathrm{O}(\mathrm{V}: \mathrm{V})=$ 10:110, $\triangle \quad \mathrm{HCl}: \mathrm{H}_{2} \mathrm{O}(\mathrm{V}: \mathrm{V})=15: 105,-\quad \mathrm{HCl}: \mathrm{H}_{2} \mathrm{O}(\mathrm{V}: \mathrm{V})=20: 100, \times \quad \mathrm{HCl}: \mathrm{H}_{2} \mathrm{O}(\mathrm{V}: \mathrm{V})=$ 40:80

The molar fraction $(x)$ of the solute can be determined using the following equation:

$$
x=\frac{m_{C B[5]} / M_{C B[5]}}{m_{C B[5]} / M_{C B[5]}+m_{H_{2} \mathrm{O}} / M_{H_{2} \mathrm{O}}+m_{H C l} / M_{H C l}}
$$

From above Table 1, and Figure1, it can be seen that the solubility of CB[5] in pure water and different concentration of hydrochloric acid aqueous solution increase with increasing temperature. It is interesting that the solubility of $\mathrm{CB}[5]$ increase with increasing acid concentration below $298 \mathrm{~K}$ and the solubility of $\mathrm{CB}[5]$ reduce with increasing acid concentration above $305 \mathrm{~K}$.As known from the literature,[13,14,17] the solubility of $\mathrm{CB}[5]$ increase with increasing acid concentration at 298.15K. For a long time, scientists maybe believe that the solubility of CB[5] increase with increasing acid concentration at any temperature, due to there is no report on the study with the solubility of $\mathrm{CB}[5]$ in different acid concentration at different temperatures. Now we know that 
there are different rules at different temperatures.

The information of Table 1 and Figure 1 indicate that the solubility of CB[5] grew exponentially in pure water and different concentration of hydrochloric acid aqueous solution. The solubility of $\mathrm{CB}[5]$ with changing temperature can be correlated by the modified Apelblat equation [26], due to that $\ln x$ demonstrate a linear relationship with the reciprocal of the absolute temperature. The modified Apelblat equation is

$\ln x=A+\frac{B}{T / K}+C \ln (T / K)$

where $x$ is the molar fraction of solubility of CB[5], $T$ is the absolute temperature(K), $A, B$ and $C$ are the parameters of the exponential expression.

As known from the reference, the dissolution enthalpy for a nonideal solution can be obtained from the modified van't Hoff equation. So the dissolution enthalpy $\left(\Delta_{\text {dis }} H\right)$ and entropy $\left(\Delta_{\text {dis }} S\right)$ for $\mathrm{CB}[5]$ was calculated by eq.3 base on the reference [27].

$$
\ln x=\frac{\Delta_{\text {dis }} H}{R T}+\frac{\Delta_{\text {dis }} S}{R}
$$

where $x$ is the mole fraction solubility of CB[n], $\Delta_{\text {fus }} H$ and $\Delta_{\text {fus }} S$ are, respectively, the dissolution enthalpy and dissolution entropy, $R$ is the ideal gas constant $\left(8.314510 \mathrm{~J} \cdot \mathrm{mol}^{-1} \cdot \mathrm{K}^{-1}\right)$, and $T$ is absolute temperature.

The change of Gibbs free energy $\left(\Delta_{d i s} G\right)$ can be obtained by the equation 4 and equation 5 [28-29]:

$$
\begin{aligned}
& \Delta_{\text {dis }} G=\Delta_{d i s} H-T_{m} \Delta_{d i s} S \\
& T_{m}=\frac{n}{\sum_{i=1}^{n}\left(\frac{1}{T_{i}}\right)}
\end{aligned}
$$

$T_{\mathrm{m}}$ in equation 5 stands for the mean harmonic temperature, and $\mathrm{n}$ is the number of experimental temperatures.

Table 2 listed the values of dissolution enthalpy $\left(\Delta_{\text {dis }} H\right)$, dissolution entropy $\left(\Delta_{\text {dis }} S\right)$, and the change of Gibbs free energy $\left(\Delta_{\text {dis }} G\right)$ for the dissolution of $\mathrm{CB}$ [5] in pure water and different concentration of hydrochloric acid aqueous solution.

Table 2. The values of $\Delta_{\text {dis }} H, \Delta_{\text {dis }} S, \Delta_{\text {dis }} G$ of CB[5] in different solvents

\begin{tabular}{cccc}
\hline solvent & $\Delta$ disH/J·mol-1 & $\Delta$ dis $/ \mathrm{J} \cdot \mathrm{K}-1 \cdot \mathrm{mol}-1$ & $\Delta$ disG/J·mol-1 \\
\hline $0: 120=\mathrm{HCl}: \mathrm{H}_{2} \mathrm{O}(\mathrm{V}: \mathrm{V})$ & 66686.3574 & 160.8798 & 18855.2347 \\
$10: 110=\mathrm{HCl}: \mathrm{H}_{2} \mathrm{O}(\mathrm{V}: \mathrm{V})$ & 48643.2080 & 101.0217 & 18608.4551 \\
$15: 105=\mathrm{HCl}: \mathrm{H}_{2} \mathrm{O}(\mathrm{V}: \mathrm{V})$ & 40413.6349 & 73.3155 & 18616.2115 \\
$20: 100=\mathrm{HCl}: \mathrm{H}_{2} \mathrm{O}(\mathrm{V}: \mathrm{V})$ & 44396.0038 & 87.4608 & 18393.0374 \\
$40: 80=\mathrm{HCl}: \mathrm{H}_{2} \mathrm{O}(\mathrm{V}: \mathrm{V})$ & 31935.4756 & 45.2339 & 18486.9858 \\
\hline
\end{tabular}

\section{Conclusion}

In this study, the solubility of $\mathrm{CB}$ [5] in pure water and different concentration of hydrochloric acid aqueous solution under the temperature ranging from 283.15 to $323.15 \mathrm{~K}$ was measured by using UV-vis spectrophotometer method. In terms of the temperature effect, the solubility of CB[5] increased with the increasing of temperature in all of solvents studied. A new discovery from the experimental result was that the solubility of $\mathrm{CB}[5]$ increased with increasing acid concentration below $298 \mathrm{~K}$ and the solubility of $\mathrm{CB}[5]$ reduced with increasing acid concentration above $305 \mathrm{~K}$. The dissolving enthalpies and entropies of CB[5] in different solvents was calculated by the Van't 
Hoff equation respectively on the basis of the experimental solubility data, and the Gibbs free energy was also calculated.

\section{Experimental Section}

Materials. In the experiments, $\mathrm{CB}[5]$ was synthesized based on the literature [30-31] and the purity of CB[5] was more than $99.5 \%$ in mass. The deionized water $\left(18.25 \mathrm{M} \Omega \cdot \mathrm{cm}^{-1}\right)$ was obtained from a Millipore Mili-Q Plus water system, and the hydrochloric acid was purchased from Beijing Chemical Reagent Co. without further purification. A UV-4802S spectrophotometer was supplied by UNICO (Shanghai) Instrument Co., Ltd.

Sample Preparation. The solvents $(10 \mathrm{~mL})$ and excess amount of CB[5] was added into glass vials, and then the vials were heated in a temperature thermostatic reaction bath with an uncertainty of $\pm 0.1 \mathrm{~K}$. The mixture solution was stirred with a magnetic stir bar at the speed of $800 \mathrm{rpm}$ continuously for 4 hours at the constant temperature. After the solution equilibrium was attained, the suspensions were settled at constant temperature for $4 \mathrm{~h}$. For each sample, $0.1 \mathrm{~mL}$ of the supernatant liquid was transferred into glass bottles by Pipette, then the solvent was evaporated at room temperature and pressure. After the evaporation of the solvent, the residue was dried in vacuum and dissolved in pure water. Then the solution was transferred into a volumetric flask completely and diluted to an appropriate concentration. The absorbance of the diluted solutions was measured on a UV-4802S spectrophotometer at room temperature. All of the experiments were repeated 3 times at each temperature.

Sample Analysis. To obtain the CB[5] concentration in different solutions, the absorbance of the standards and samples were measured at $197 \mathrm{~nm}$, which is the best absorption wavelength of CB[5] as determined by us, using pure water as a reference [32]. The standard curve equation for the determination of $\mathrm{CB}[5]$ was obtained as $y=0.1548 x-0.0374$ ( $y$ was UV corresponding absorbance and $x$ was concentration of standard solutions) with the correlation coefficient $R^{2}=0.9998$ in the concentration ranging from $0.0234 \mathrm{~g} \cdot \mathrm{L}^{-1}\left(2.819277 \cdot 10^{-5} \mathrm{~mol} \cdot \mathrm{L}^{-1}\right)$ to $0.0702 \mathrm{~g} \cdot \mathrm{L}^{-1}\left(8.457831 \cdot 10^{-5}\right.$ $\left.\mathrm{mol} \cdot \mathrm{L}^{-1}\right)$. It is plotted in Figure 2.

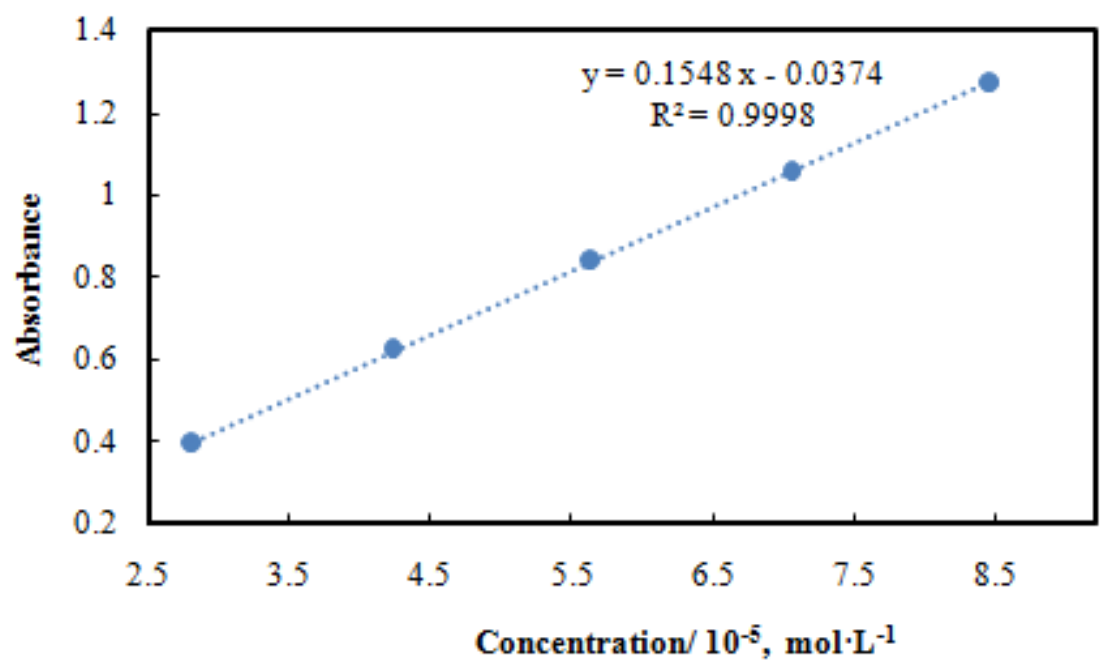

Figure 2. Standard curve of CB[5] aqueous solution

\section{Acknowledgement}

In this paper, the research was sponsored by a grant from the Beijing Institute of Technology (Grant No. 20131042006). 


\section{References}

[1] Robert Behrend, Eberhard Meyer, Franz Rusche. Liebigs Ann. Chem. 1905, 339(1), 1-37.

[2] Jaheon Kim, In-Sun Jung, Soo-Young Kim, Eunsung Lee, Jin-Koo Kang, Shigeru Sakamoto, Kentaro Yamaguchi, Kimoon Kim. J. Am. Chem. Soc. 2000, 122(3), 540-541.

[3] Anthony I. Day , Rodney J. Blanch, Alan P. Arnold, Susan Lorenzo, Gareth R. Lewis,Ian Dance. Angew. Chem. Int. Ed. 2002, 41(2), 275-277.

[4] Simin Liu, Peter Y. Zavalij, Lyle Isaacs. J. Am. Chem. Soc. 2005, 127(48), 16798-16799.

[5] Anthony Day, Alan P. Arnold, Rodney J. Blanch, Barry Snushall. J. Org. Chem. 2001, 66(24), 8094-8100.

[6] Lyle Isaacs, Sang-Kyu Park, Simin Liu, Young Ho Ko, Narayanan Selvapalam, Youngkook Kim, Hyunuk Kim, Peter Y. Zavalij, Ghyung-Hwa Kim, Heung-Soo Lee, Kimoon Kim. J. Am. Chem. Soc. 2005, 127(51), 18000-18001.

[7] Xiao-Jie Cheng, Li-Li Liang, Kai Chen, Ning-Ning Ji, Xin Xiao, Jian-Xin Zhang, Yun-Qian Zhang, Sai-Feng Xue, Qian-Jiang Zhu, Xin-Long Ni, Zhu Tao. Angew. Chem. Int. Ed. 2013, 52(28), 7252-7255.

[8] Wade. A. Freeman, William. L. Mock, Neng-Yao Shih. J. Am. Chem. Soc. 1981, 103(24), 7367-7368.

[9] Vanya D. Uzunova, Carleen Cullinane, Klaudia Brix, Werner M. Nau, Anthony I. Day. Org. Biomol. Chem. 2010, 8, 2037-2042.

[10] Young Ho Ko, Eunju Kim, Ilha Hwanga, Kimoon Kim. Chem. Commun. 2007, 13, 1305-1315.

[11] Garima Ghale, Vijayakumar Ramalingam, Adam R. Urbach, Werner M. Nau. J. Am. Chem. Soc. 2011, 133(19), 7528-7535

[12] Hyunuk Kim, Yonghwi Kim, Minyoung Yoon, Soyoung Lim, Se Min Park, Gon Seo, Kimoon Kim. J. Am. Chem. Soc., 2010, 132(35), 12200-12202.

[13] Guiling Zhang, Guoqing Xu, Saifeng Xue, Qianjiang Zhu, Zhu Tao. Chinese J. Inorg. Chem. 2003, 19(6), 655-659.

[14] Hans-Jürgen Buschmann, Klaus Jansen, Claudia Meschke, Eckhard Schollmeyer. J. Solution Chem. 1998, 27(2), 135-140.

[15] Silke Karcher, Anja Kornmuller, Martin Jekel. Wat. Res. 2001, 35(14), 3309-3316.

[16] Klaus Jansen, Hans-Jürgen Buschmann, Andreas Wego, Dietrich Döpp, Colin Mayer, Hans-Joachim Drexler, Hans-Jürgen Holdt, Eckhard Schollmeyer. J. Inclusion Phenom. Macrocyclic Chem. 2001, 39(3), 357-363.

[17] Hans-Jürgen Buschmann, Ernst Cleve, Klaus Jansen, Andreas Wego, Eckhard Schollmeyer. J. Inclusion Phenom. Macrocyclic Chem. 2001, 40(1), 117-120.

[18] Guiling Zhang, Liang Qu, Xuqiang Luo, Jia Zeng. Chemistry Online 2014, 77(6), 515-520.

[19] Petr Toman Emanuel Makrlı'k Petr Vanura. Monatshefte fuer Chemie 2013, 144(6), 813-816.

[20] Qian Wang, Saifeng Xue, Kai Chen, Zhu Tao. Chinese J. Inorg. Chem. 2011, 27(3), 463-467.

[21] S. Kushwaha, Srinivas A. Rao , Padmaja P. Sudhakar. Inorg. Chem. 2012, 51(1), 267-273.

[22] Li-Li Liang, Kai Chen, Xing Feng, Yun-Qian Zhang, Qian-Jiang Zhu, Sai-Feng Xue, Zhu Tao. 
J. Mol. Struct. 2011, 1006(1), 87-90.

[23] Kai Chen, Li-Li Liang, Hao-Jing Liu, Yun-Qian Zhang, Sai-Feng Xue, Zhu Tao, Xin Xiao, Qian-Jiang Zhu, Leonard F. Lindoy, Gang Wei. CrystEngComm 2012, 14(23), 7994-7999

[24] Zhang Guiling; Luo Xuqiang; Ran Liang; Zeng Jia. Computers and Applied Chemistry. 2014, 31(1), 55-58.

[25] Werner Nau, Jyotirmayee Mohanty. US 20080023646.

[26] Jyothi Thati , Fredrik L. Nordström, Åke C. Rasmuson. J. Chem. Eng. Data 2010, 55(11), 5124-5127.

[27] Kejian Cui, Zhibin Xu, Liru Chen, Min Xue, Zihui Meng, Guangrui Xu, Rui Wang, Wenjin Liu, and Zhihui Lin. J. Chem. Eng. Data 2013, 58(9), 2677-2680.

[28] Hui Wang, Qinbo Wang, Zhenhua Xiong, Chuxiong Chen, Binwei Shen. J. Chem. Thermodynamics 2015, 83, 61-66.

[29] Rongrong Li, Hao Chen, Huayue Zhu, Ru Jiang, Stephen Louis Romano, Xiaoying Chen, Deman Han. J. Chem. Thermodynamics 2015, 83, 1-5.

[30] Fei Meng. M.S. Thesis, Guizhou University, 2006, 18-20.

[31] Qiang Bi, Ying-peng Hu, Qin Yang, Cai-lian Ma, Dongliang Li. Chin. J. Org. Chem. 2007, 27(7), 880-884.

[32] Longxiao Liu, Jun Chen. J. Chem. Eng. Data 2008, 53(7), 1649-1650. 